\title{
An Impact of the Particle Size Distribution on CP Degree at the Mechanism of Shear-Induced Diffusion in Cross-Flow Microfiltration
}

\author{
Sergey P. Agashichev*
}

\author{
NEWRC (ADWEA), PO Box 54111, Abu Dhabi, UAE
}

\begin{abstract}
Model for concentration profile at the mechanism of shear- induced back migration has been proposed. The model is based on the following assumptions: (1) Flow is assumed to be isothermal, incompressible, fully developed and steady- state. The model implies the position dependence of transverse velocity and shear rate; (2) Dispersed phase consists of rigid bodies characterized by identity variables and particle- size distribution; (3) Back migration being nondiffusive in nature is characterized by certain kinetic behavior. The migration of particle is determined by shear- induced force (moving force) and drag forces (resistance force); (4) The migration of particles was assumed to occur within the zone referred to as shear-diffusion migration zone. Migration zone ranges from membrane to its threshold value at the upper boundary where the state of pseudo-equilibrium takes place; Vector sum of the forces acting the particle (shear and drag forces) ranges from its maximum value at the surface of membrane to zero at the upper boundary of migration zone where the state of pseudo-equilibrium takes place; (5) The driving force being degree of deviation from the state of equilibrium was expressed as the difference between local shear rate and its threshold value at the upper boundary of migration zone; Transport mechanisms such as Brownian diffusion, inertial lift, interaction based on Van der Waals or electrostatic forces, combined effects of particle-particle and particle-membrane interactions are outside the scope of the model. The proposed model has the following possible implications: (A) It can be used for quantitative estimation of the probability of fouling caused by different fractions at existing hydrodynamic conditions; (B) The model allows analyzing the impact of hydrodynamic conditions (such as shear stress and transverse velocity) on the probability of fouling caused by certain dispersed fraction; (C) It allows estimation of the threshold value of the shear rate specific for the certain fraction that should be exceed in order to prevent accumulation of this fraction; (D) Proposed model permits analyzing the influence of shear rate at the surface of membrane on the transverse concentration distribution; (E) The model can be used for quantitative analysis of CP distribution of individual fraction while considering poly-disperse systems as soon as any fraction is characterized by its individual migrating behavior.
\end{abstract}

Keywords: Shear-induced diffusion, concentration polarization, cross-flow microfiltration, modeling.

\section{INTRODUCTION, FORMULATION OF THE PROBLEM AND OBJECTIVES OF THE STUDY}

Pressure- driven processes are inevitably accompanied by the phenomena such as concentration polarization and membrane fouling. As soon as the feed solution contains multiple fouling factors such as suspended matter, solvable organics, colloids, etc., characterized by different physical behavior and various transport mechanism, it's getting essential to quantify the contribution of individual transport mechanism. There are following mechanisms of back migration: Brownian diffusion, shear-induced migration or inertial lift. Brownian diffusion is a classical transport mechanism where the diffusion coefficient can be estimated from the Stokes-Einstein relationship. However, the permeate flux predicted by the classical Brownian concept is one or more orders of magnitude below experimental data. For these phenomena to be explained Zydney and Colton [1,2] proposed new CP model that assumes migration of particles in the directions of the lower shear rate, where the diffusion

*Address correspondence to this author at the NEWRC (ADWEA), PO Box 54111, Abu Dhabi, UAE; Tel: +971506612671;

E-mail: agashichev@gmail.com coefficient was expressed as $D=0.03 r^{2} \dot{\gamma}_{\text {wall }}$ where $r-$ radius of particle; $\dot{\gamma}_{\text {wall }}$ - wall shear rate. This gave improved agreement with the experiments. This transport phenomenon was referred to as "shearinduced diffusion" that can be considered as dominant transport mechanism at cross -flow filtration at elevated tangential velocity for particles in the range $0.5-30 \mu \mathrm{m}$, [3]. That model was further developed by Romero, Davis and Sherwood [4- 7] for a dilute solution. This mechanism has been intensively scrutinized by different authors, [8-13]. Back transport for particles greater than $10 \mu \mathrm{m}$ in radius is controlled by so called inertial lift mechanism. It was considered by Belfort and co-authors [14,15]. Inertial lift involves a lateral migration of particles away from the membrane, it arises from hydrodynamic interactions associated with the distortion of the fluid streamlines in the gap between the particle and the flow boundary, [14]. The inertial lift velocity is proportional to the cube of the particle radius and the square of the local shear rate. It dominates over the shear-induced diffusion for particles greater than $10 \mu \mathrm{m}$ in radius. The conditions where the certain mechanism prevails were considered by Robert van Reis and Andrew Zydney in [16]. According to the study done by $\mathrm{T}$. Jiang et al. [17] submicron particles were likely to deposit in side-stream MBRs, and the lowest back transport velocity was found for particle radii around $0.1 \mu \mathrm{m}$ and cross flow below $0.5 \mathrm{~m} / \mathrm{s}$. A 
particle size distribution profile of MBR sludge showed the main peak at $40 \mu \mathrm{m}$ and second peak at $0.1-1 \mu \mathrm{m}$. In cases when the permeation velocity exceeds the back-transport velocity the particles have a higher likelihood to deposit. The critical particle size, on which the permeation and back-transport velocity are balanced, at $U=1 \mathrm{~m} / \mathrm{s}$ is $1.5 \mu \mathrm{m}$. Increasing the crossflow velocity up to $4 \mathrm{~m} / \mathrm{s}$ is able to reduce the critical particle size down to $0.3 \mu \mathrm{m}$. On the other hand, for particles larger than $10 \mu \mathrm{m}$, even very low cross-flow velocities $(0.3 \mathrm{~m} / \mathrm{s})$ can keep them in_suspension, [17]. According to [18] the mean diameter of deposited particles decayed to $5.5 \mu \mathrm{m}$ at a fluid velocity of $0.23 \mathrm{~m} / \mathrm{s}$ and a flux of $15 \mathrm{I} / \mathrm{h}-\mathrm{m} 2$, and to $5.0 \mu \mathrm{m}$ at a speed of $3 \mathrm{~m} / \mathrm{s}$ and a flux of $60 \mathrm{l} / \mathrm{h}-\mathrm{m} 2$. Analyses done by [19] revealed the particle- size distribution of the deposited particles at different cross flow velocities. It was demonstrated that the particles in the cake layer are smaller at higher cross flow velocity [19] The estimated velocity of the particle as it moved across the surface was $0.3 \mathrm{~mm} / \mathrm{s}$; cross flow velocity was approximately $0.05 \mathrm{~m} / \mathrm{s}$. Comparison of the size of deposited particles at different cross flow velocity was submitted in [19]. Recent studies reported that particleparticle and particle-membrane interactions (including entropy, van der Waals interactions and electrostatic interactions) may also play an essential role in transport especially in concentrated solutions of the colloids $[20,21]$. The study by Mungman and Zydney [20] focused on analysis of crow-flow filtration using numerical integration of the Langevin equation accounting for combined effects of electrostatic repulsion, enhanced hydrodynamic drag, Brownian diffusion, inertial lift and van der Waals attraction. According to [20] the critical flux increases with increases of shear rate, although this effect doesn't become significant until $\dot{\gamma}_{m}=12,000 \mathrm{~s}-1$ for $\mathrm{a}=1 \mathrm{~nm}$, and $\dot{\gamma}_{m}=1000 \mathrm{~s}-1$ for $a=5 \mathrm{~nm}$. The critical flux increases with increasing the particle radius due to the greater electrostatic repulsion between membrane and the larger particles. According to [21] 10\% of publications related to fouling are focuses on the critical flux concept. Since recently one can see the growth of publications focused on quantitative relation between shear- induced diffusion, critical flux and particle- size distribution in liquid phase and within deposited layer. Further development of the existing models allowed establishing quantitative links between the variables of shear induced transport such as the size or particle, shear rate, transverse velocity and the variables specifying the critical flux and probability of the fouling rate for certain dispersed fractions, [17-25]. It was experimentally demonstrated and confirmed by numerical studies that the stochastic motion of the particles is not diffusive all the times and the particles tend to accumulate in regions of low shear rate. Summarizing the data [16-26] the following can be concluded: (1) the critical flux increases with increasing the particle radius; (2) submicron particles demonstrate higher likelihood to deposit and the distribution spectrum of cake particles was shifted towards smaller diameter; (3) analyses of the size distribution of the deposited particles at different cross flow velocities conducted by [19] revealed that the particle size in the cake layer are smaller at higher cross flow velocity; (4) conventional mathematical formulations of the degree of concentration polarization based on mass concentration cannot be applied for the case of polydispersed suspension since any fraction is characterized by individual distribution curve depending upon applied shear rate. In this regard this study represents an attempt to propose relation between the concentration distributions within liquid phase and shear rate depending upon the size of particle in the case if the shear-induced migration takes place.

\section{PHYSICAL ASPECTS AND ASSUMPTION UNDERLYING THE MODEL}

The system consists of non- interacting hard spheres being dispersed in liquid phase. Some transport mechanisms such as Brownian diffusion and inertial lift along with certain mechanisms of interaction based on Van der Waals or electrostatic forces and combined effects of particle-particle and particle membrane interactions are outside the scope of the model. The model is based on the concepts of critical velocity and shear-induced back migration. The range of particle size, the model can be applied, covers 1- 5 microns.

\subsection{Main Assumptions}

The fluid is assumed to be continuous, noncompressible and isothermal at steady state

Longitudinal velocity is approximated by parabolic function as

$u(\theta)=U_{\text {MAX }}\left(1-\theta^{2}\right)$

It ranges form $u(\theta)=U_{\text {MAX }}$ at the centerline to $u(\theta)=0$ at the membrane. There is no slip at membrane surface.

Transverse velocity, $V(\theta)$, was based on the Berman's approximation, [27].

$V(\theta)=V_{\text {MAX }}\left[\frac{\theta}{2}\left(3-\theta^{2}\right)\right]$

It ranges form $V(\theta)=0$ at the centerline to its maximum value $V(\theta)=V_{\operatorname{MAX}(\theta=0)}$ at the surface of membrane 
Shear-rate profile, $\dot{\gamma}(\theta)=d u(\theta) / d \theta$, in terms of the $\theta$ variable gives

$\dot{\gamma}(\theta)=\dot{\gamma}_{M A X(\theta=1)} \theta$

It ranges form $\dot{\gamma}(\theta)=0$ at the centerline to its maximum value $\dot{\gamma}_{M A X(\theta=1)}=2 U_{M A X} / H$ at the surface of membrane

Shear stress profile, $\tau(\theta)=-\mu(d u / d \theta)$ in terms of the $\theta$ variable can be expresses as

$\tau(\theta)=\mu \frac{2 U_{M A X}}{H} \theta$

Behavior of hydrodynamic characteristics such as $u(\theta), V(\theta)$ and $\tau(\theta)$ is shown in Figure $\mathbf{1}$.

\subsection{Forces Acting the Particles}

The back migration of dispersed phase being nondiffusive in nature can be characterized by certain kinetic behavior. The main forces exerted on the particle are the shear-induced force and drag (resistance) forces. The resultant force $\bar{F}_{\Sigma}$ the migration influenced is the vector sum

$\bar{F}_{\Sigma}=\vec{F}_{\text {shear }}+\vec{F}_{\text {drag }}$
Where the shear generated force acting the dispersed particle is proportional to shear stress (and in turn to shear rate) that gives the resultant towards centerline.

$\vec{F}_{\text {shear }}=\oint \bar{\tau} d A$

Where $\vec{F}_{\text {shear }}$ net shear force in Z- direction; $\bar{\tau}$ - shear stress vector pointing towards the transverse flow direction. Shear force can be expressed as

$$
F_{\text {shear }}=\mu \Delta \dot{\gamma}(\theta) A
$$

where $A=4 \pi R^{2}$ - surface of the particle with the radius$\mathrm{R} ; \mu$-dynamic viscosity; $\Delta \dot{\gamma}(\theta)$ - difference between the current value of the shear rate and the value at upper boundary of the migration zone, $\Delta \dot{\gamma}(\theta)=\dot{\gamma}_{\max }\left(\theta-\theta_{\text {crit }}\right)$. The shear force ranges from its maximum value at membrane $F_{\text {shear }(\max )}=F_{(\theta=1)}$ to $F_{\text {shear }}=0$ at $\theta=\theta_{\text {crit }}$

Drag force based on the Stokes Law can be written as

$F_{d r a g}=6 \pi \mu R[W(\theta)+V(\theta)]$

Where $V(\theta)$ - transverse liquid velocity; $W(\theta)$ velocity of the back migration (relative to membrane

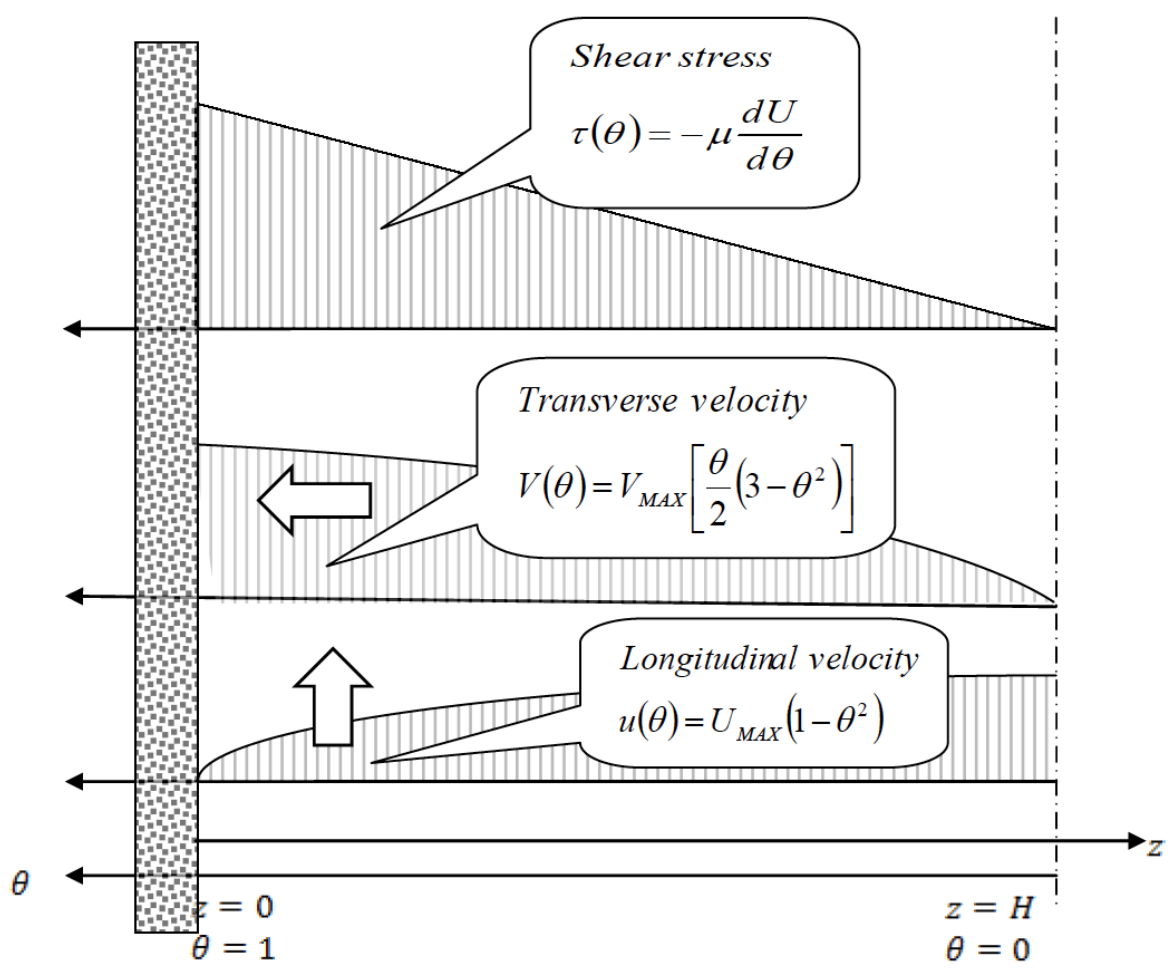

Figure 1: Shear stress, longitudinal and transverse velocity distribution. 


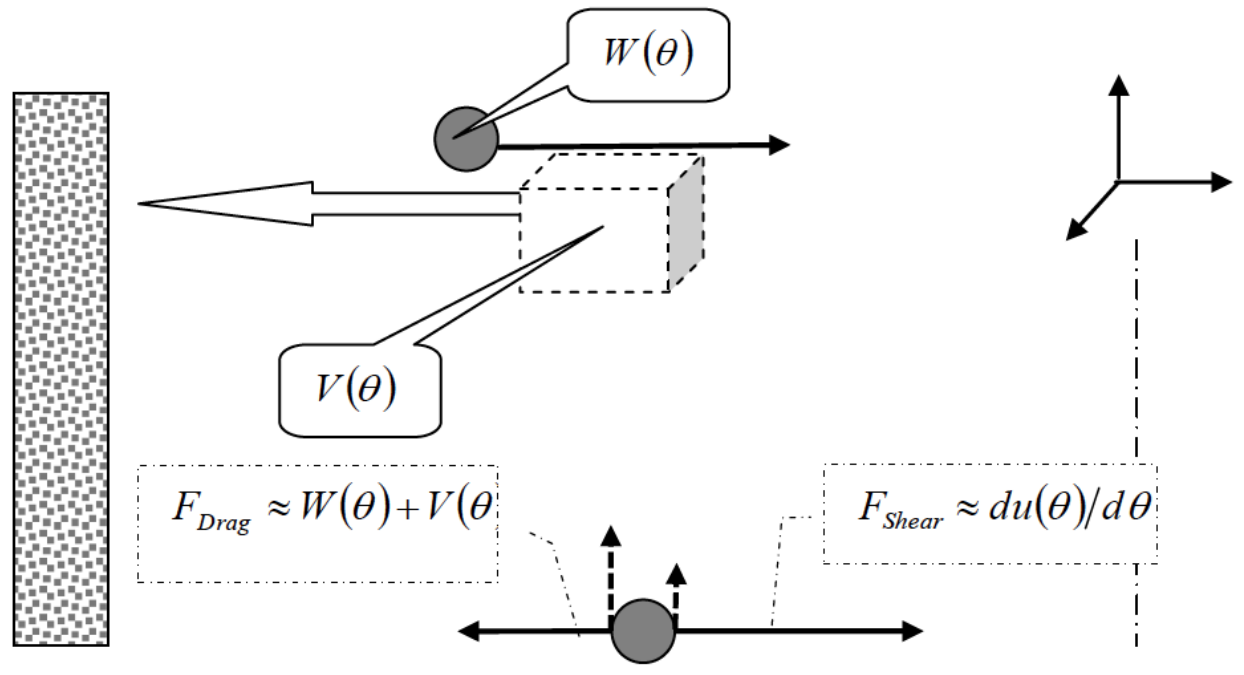

Figure 2: Velocity of back migration and the main forces acting the particle.

surface). The drag force varies from $F_{\operatorname{drag}(\theta=1)}$, at membrane to certain value at the upper boundary of the migration zone at $\theta=\theta_{\text {crit }}$, where $F_{\text {shear }}\left(\theta_{\text {crit }}\right)=F_{\text {drag }}\left(\theta_{\text {crit }}\right)$

\subsection{Migration Zone}

As soon as the resultant force acting the particle (vector sum of the shear and drag forces) ranges from its maximum value at the membrane surface to zero at the distance where the state of pseudo-equilibrium occurs. The migration of suspended phase can be observed within this zone that can be referred to as the migration zone (or shear-diffusion zone). It ranges from membrane surface to its threshold value (or the upper boundary) at $\theta_{\text {crit }}$ where $F_{\text {shear }}=F_{\text {drag }}$ The velocity of back migration at the upper boundary of the zone has to be zero, $W\left(\theta_{\text {crit }}\right)=0$ The distance of potential migration of any fraction $r_{i}$ is characterized by its individual threshold value or the upper boundary of migration zone- $\theta_{\text {crit }}\left(r_{i}\right)$. According to published data the bigger size of the particles the more prolonged the corridor of migration thus the more distant the upper boundary of the migration zone. At the upper boundary the state of pseudo-equilibrium is assumed to take place (at $F_{\text {shear }}=F_{\text {drag }}$ ). The upper boundary of the migration zone is characterized by equality between the local shear stress and its threshold value for particular fraction, $\theta_{\text {crit }}=\dot{\gamma}_{\text {crit }} / \dot{\gamma}_{(\theta=1)}$

\subsection{Driving Force of Back Migration}

As soon as the model of physical behavior assumes that the migration of dispersed particle can take place under the conditions if the shear stress exceeds its threshold (or critical) value. Once the back migration was assumed to be driven by the shear stress difference, the driving force $\Delta \dot{\gamma}(\theta)$ (being a degree of deviation from the equilibrium state) can be expressed as the difference between local shear rate and its threshold value. The threshold value in turn represents the shear rate at the threshold (critical) distance $\theta_{\text {crit }}$ where the state of pseudo-equilibrium takes place (see Figure 3).

$\Delta \dot{\gamma}(\theta)=\dot{\gamma}(\theta)-\dot{\gamma}\left(\theta_{\text {crit }}\right)$

The second term on the right hand side of Eq. (10) represents the threshold value of the shear rate $\dot{\gamma}\left(\theta_{\text {crit }}\right)$ at the critical distance, $\theta_{\text {crit }}$. The driving force ranges from its maximum value at membrane being equal to $\Delta \dot{\gamma}_{\max }(\theta)=\left(\dot{\gamma}_{\max (\theta=1)}-\dot{\gamma}_{\text {crit }}\right)$ to $\Delta \dot{\gamma}(\theta)=0$ at the critical distance $\theta_{\text {crit }}=1-\dot{\gamma}_{\text {crit }} / \dot{\gamma}_{\max (\theta=0)}$. Similarly it can be expressed in terms of the shear stress as follows

$\Delta \tau(\theta)=\mu \dot{\gamma}(\theta)-\mu \dot{\gamma}_{\text {crit }}\left(\theta_{\text {crit }}\right)$

\section{IMPACT OF THE PARTICLE SIZE AND SHEAR STRESS ON THE UPPER BOUNDARY OF MIGRATION ZONE}

The migration zone ranges from $\theta=1$ at the membrane surface to $\theta=\theta_{\text {crit }}$ at the upper boundary where the state of pseudo-equilibrium was assumed to occur, $F_{\text {shear }}=F_{\text {drag }}$. Migration behavior depends upon the shear force and drag (resistance) forces. The vector sum of these forces reaches zero at the upper boundary where the net velocity vector is assumed to be zero as well, $w^{*}(\theta)=0$. (The coordinate origin at the membrane surface). Similarly if the coordinate origin is 


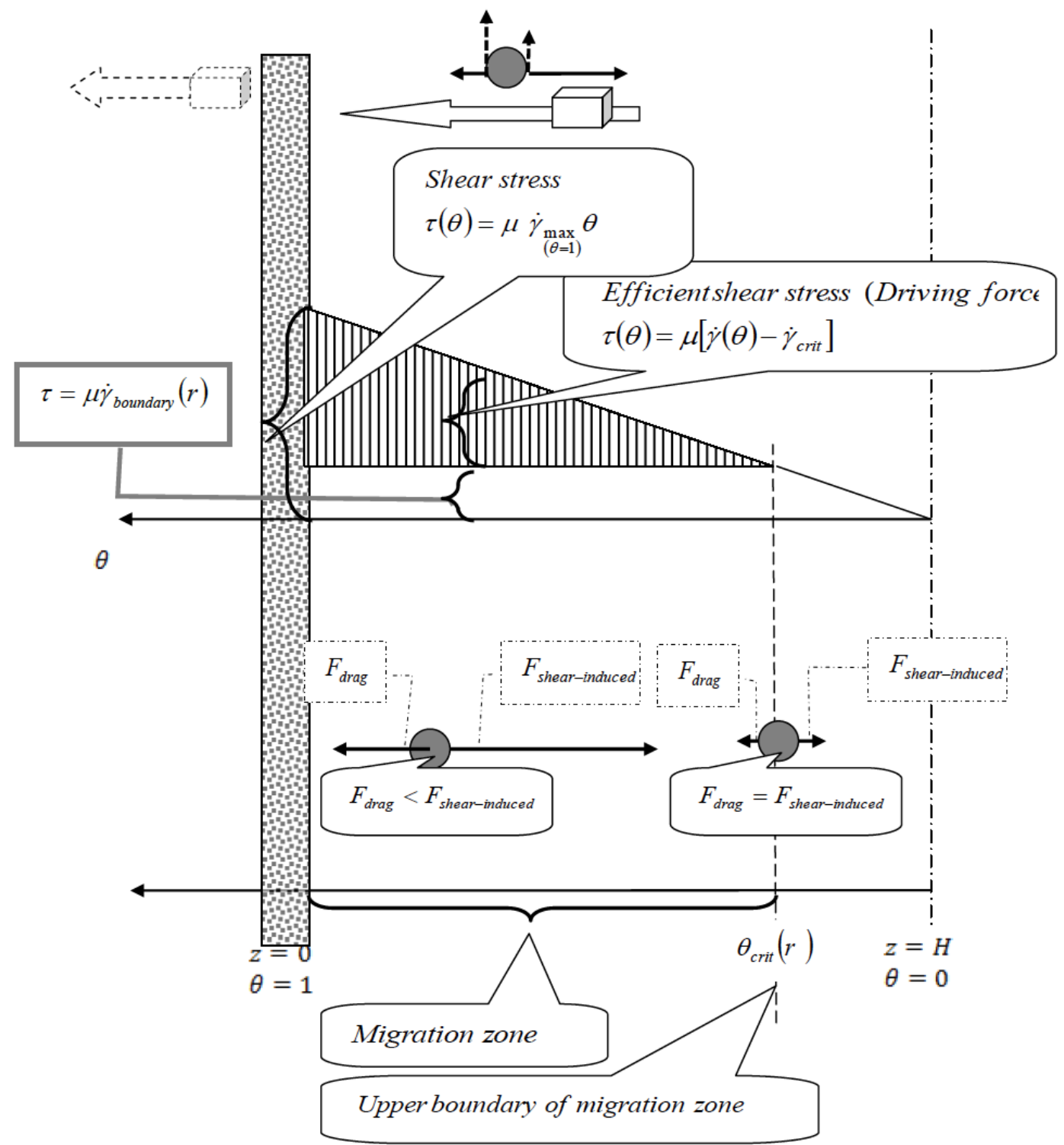

Figure 3: Shear- induced transport within the migration zone.

at the particle it gives $w(\theta)=-v(\theta)$. Inserting Berman's distribution for transverse velocity into (9) we get $F_{\text {drag }}$ at $\theta=\theta_{\text {crit }}$.

$$
\underset{\substack{\text { drag } \\\left(\theta_{-} \text {crit }\right)}}{F_{\text {a }}}=6 \pi \mu R V_{\text {MAX }} \frac{\theta_{\text {crit }}}{2}\left(3-\theta_{\text {crit }}^{2}\right)
$$

Combining Eqs. (8) and (12) we get

$\mu \dot{\gamma}\left(\theta_{\text {crit }}\right) 4 \pi R^{2}=6 \pi \mu R V_{\text {MAX }} \frac{\theta_{\text {crit }}}{2}\left(3-\theta_{\text {crit }}^{2}\right)$

Having been rearranged Eq. (13) gives the size of dispersed fraction-R; vs. upper boundary of migration zone- $\theta_{\text {crit }}$; shear rate and transverse velocity at membrane surface, $\dot{\gamma}_{\max }$ and $V_{\max }$ respectively.
$R=3 V_{\max }\left(3-\theta_{\text {crit }}^{2}\right) / 4 \dot{\gamma}_{\max }$

Boundary conditions:

At membrane surface $\theta=1$

$R=3 V_{\text {max }} / 2 \dot{\gamma}_{\text {max }}=1.5 V_{\text {max }} / \dot{\gamma}_{\text {max }}$

At centerline where $\theta=0$

$R=9 V_{\max } / 4 \dot{\gamma}_{\max }=2.25 V_{\max } / \dot{\gamma}_{\text {max }}$

Eq. (14) gives the coordinate of upper boundary of migration zone

$\theta_{\text {crit }}=\sqrt{3-4 \dot{\gamma}_{\max } R / 3 V_{\max }}$

Set of calculated values of the upper boundary vs. size of fraction based on Eq. (17) is shown in Figure 4. 


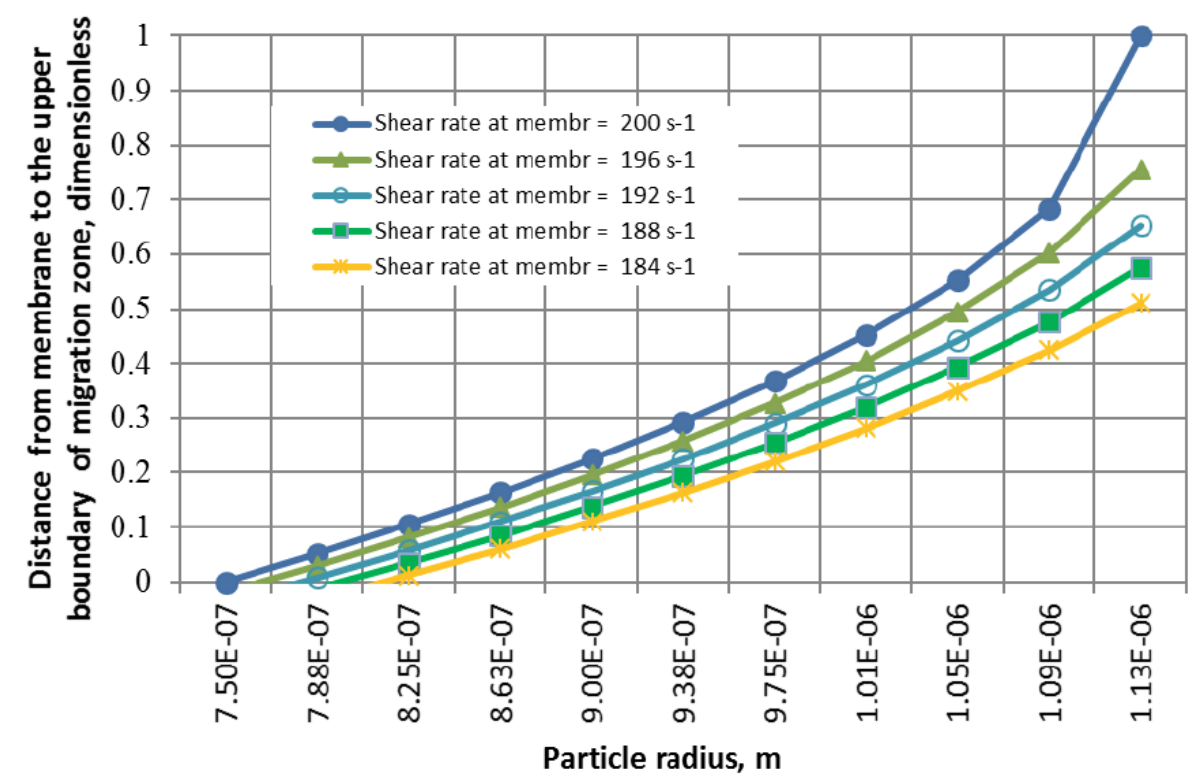

Figure 4: Impact of the particle size -R on the upper boundary of migration zone- $\theta_{\text {crit }}$ at arbitrary values of the shear rate at membrane surface, $\mathrm{V}_{\mathrm{MAX}}=1.0 \mathrm{E}-04 \mathrm{~m} / \mathrm{s}$ (Model).

It's shown that the bigger size of the fraction the further distance between membrane and equilibrium distance (or upper boundary of migration zone).

Physically the fouling can be interpreted as a phenomenon where the vector sum of shear and drag forces is equal to zero at membrane surface, $F_{\text {shear }(\theta=1)}=F_{\operatorname{drag}(\theta=1)}$. In this case the upper boundary of migration zone belongs to membrane surface, $\theta_{\text {crit }}=1$. The ratio of shear to drag forces at the membrane can be used for estimation of the probability of fouling formation, $f(R)$ by arbitrary fraction with certain Rradius as $f(R) \approx F_{\text {shear }(\theta=1)} / F_{\text {drag }(\theta=1)}$. Using the equations for shear and drag forces (14 and 17 respectively) it gives.

$f(R) \approx R\left[\dot{\gamma}_{M A X(\theta=1)} / V_{M A X(\theta=1)}\right]$

This ratio can be applied for estimation of the impact of hydrodynamic conditions [such as shear stress and transverse velocity] on the probability of fouling due to considered fraction. The shear rate should exceed its threshold value specific for the fraction in order to prevent accumulation of the considered $R$-fraction at the surface of membrane.

$\dot{\gamma}_{M A X(\theta=1)} \geq f(R) V_{M A X(\theta=1)} / R$

Experimental data [24] on critical flux, longitudinal velocity and size of particles were used for verification of the model. Diluted latex suspension $(C=0.01 \%)$ data for different particle size were selected: (A) $V_{\text {critical }}=45$ $\mathrm{l} / \mathrm{m} 2-\mathrm{h}$ for the size $3.0 \mu \mathrm{m}$, and (B): $V_{\text {critical }}=70 \mathrm{l} / \mathrm{m} 2-\mathrm{h}$ for the size $5.0 \mu \mathrm{m}$ at cross flow $0.2 \mathrm{~m} / \mathrm{s}$. The dimensionless ratio- $f(R)$ see Eq. (18), was used for comparison between the model generated values and experimental results

$$
\left[f_{A}^{\text {model } l}(R)-f_{B}^{\text {model }}(R)\right] f_{A}^{\text {exp }}(R) /\left[f_{A}^{\exp }(R)-f_{B}^{\text {exp }}(R)\right] f_{A}^{\text {model } l}(R)=\Delta
$$

Comparing the dimensionless ratio- $\Delta$ for experimental conditions (cases A and B) with the calculated data the dimensionless difference doesn't exceed $\Delta \approx 7 \%$. The range of particles along with the level of concentrations and type of the latex selected for comparison confirm the validity of physical assumptions such as non- interacting spheres ignoring van del Waals and electrostatic forces.

\section{CONCENTRATION DISTRIBUTION OF MONO- FRACTION WITHIN THE MIGRATION ZONE}

Conventional mathematical formulation of the degree of concentration polarization (in terms of mass concentration) cannot be applied for analysis of the poly-dispersed systems as soon as any fraction is characterized by individual migration behavior and boundary of migration zone.

Any poly-disperse system can be represented as a stack of fractions $r_{j}$ with individual parameters and migrating behavior characterized by individual CP 
distribution, $c_{r j}(z)$. Mass concentration $c\left(r_{j}\right)$ of any mono-fraction- $r_{j}$ is directly proportional to the number of particles $n\left(r_{j}\right)$. Overall probability of distribution of individual fractions within the migration zone is assumed to be $100 \%$.

$\int_{\theta=1}^{\theta_{-} \text {crit }(r j)} k_{r j} c_{r j}(z) d z=1$

Further consideration will be applied for individual dispersed fraction- $r_{j}$ characterized by individual CP profile, $c_{r j}(z)$. Concentration increment $d c$ over the elementary distance $d z$ can be expressed as.

$d c(z)=-K \Delta \dot{\gamma}(z) d z$

where $\Delta \dot{\gamma}(\theta)$ is the driving force, it can be expressed as the difference between the local shear rate and its threshold value $\dot{\gamma}\left(Z_{\text {crit }}\right)$ as

$\Delta \dot{\gamma}(z)=\dot{\gamma}(z)-\dot{\gamma}\left(Z_{c r i t}\right)$

Therefore eq. (22) gives

$d c(z)=-K\left[\dot{\gamma}(z)-\dot{\gamma}\left(z_{c r i t}\right)\right] d z$

Assuming linear distribution of the shear rate $\dot{\gamma}(\theta)=\dot{\gamma}_{\max } \theta$ and using dimensionless variables $d \theta=-d z / H$, Eq. (24) gives

$d c(\theta)=-K\left[\dot{\gamma}(\theta)-\dot{\gamma}\left(\theta_{c r i t}\right)\right] d \theta$

Further rearrangements enable to write

$\int d c=K H \dot{\gamma}_{\max } \int\left(\theta-\theta_{c r i t}\right) d \theta$

Having been integrated $\mathrm{Eq}(26)$ gives

$c(\theta)=-K \dot{\gamma}_{\max }\left(\theta^{2} / 2-\theta_{\text {crit }-r j} \theta\right)+$ const

The constant of integration, const, and proportionality factor, $\mathrm{K}$, were evaluated at the following boundary conditions:

at the membrane surface

$C_{\theta=1}=C_{m e m}$

at the upper boundary of the migration zone $C_{\theta=\theta_{\text {crit }}}=C_{\max }$
$K=\frac{2\left(C_{\max }-C_{m e m b r}\right)}{\dot{\gamma}_{\max }\left(\theta_{c r i t}-1\right)^{2}}$

const $=C_{\text {mem }}+\frac{\left(C_{\max }-C_{\text {membr }}\right)}{\left(\theta_{\text {crit }}-1\right)^{2}}\left(1-2 \theta_{\text {crit }}\right)$

Inserting the constants (eqs.28 and 29) into Eq. (27) gives the concentration distribution

$c(\theta)=C_{\text {membr }}+\frac{\left(C_{\max }-C_{\text {membr }}\right)}{\left(\theta_{\text {crit }}-1\right)^{2}}\left[\theta^{2}-1-2 \theta_{\text {crit }}(\theta-1)\right]$

Eq.(30) can be expressed as dimensionless function, $c^{*}(\theta)$

$c^{*}(\theta)=\frac{c(\theta)-C_{\min }(\theta=1)}{C_{\max }\left(\theta_{\text {crit }}\right)-C_{\text {min }}(\theta=1)}=\frac{\left(2 \theta_{\text {crit }}-1-\theta\right)}{\left(\theta_{\text {crit }}-1\right)^{2}}(\theta-1)$

$c^{*}(\theta)$ ranges from $c^{*}(\theta)=0$ at the membrane to $c^{*}(\theta)=1$ at the upper boundary of migration zone. Solving eq. (31) for $c(\theta)$ we get the formulation in physical dimension.

$c(\theta)=C_{\min }(\theta=1)+c^{*}(\theta)\left[C_{\max }\left(\theta_{\text {crit }}\right)-C_{\min }(\theta=1)\right]$

Eq. (32) matches the boundary conditions. It gives $c(\theta=1)=C_{\min }$ at membrane and $c\left(\theta_{\text {crit }}\right)=C_{\max }$ at the upper boundary of migration zone.

Set of calculated dimensionless profiles at different values of the upper boundary are shown in Figure $\mathbf{5}$. (The calculations are based on eq. 32).

\section{PRACTICAL IMPLICATION OF THE MODEL}

This model has a numerous potential applications. The model can be segmented into software for the design, analysis and operation of cross- flow microfiltration. In particular it can be adopted for analysis of pilot data on MF of poly-dispersed system with individual migration behavior of particular fraction. The model can provide a quantitative relation between hydrodynamic conditions, fouling resistance and expected particle-size distribution at membrane surface.

The model can be applied for quantitative estimation of characteristics and analysis of the process behavior. The model- based results confirm the published experimental data. The model demonstrated that the smaller particle size the higher 


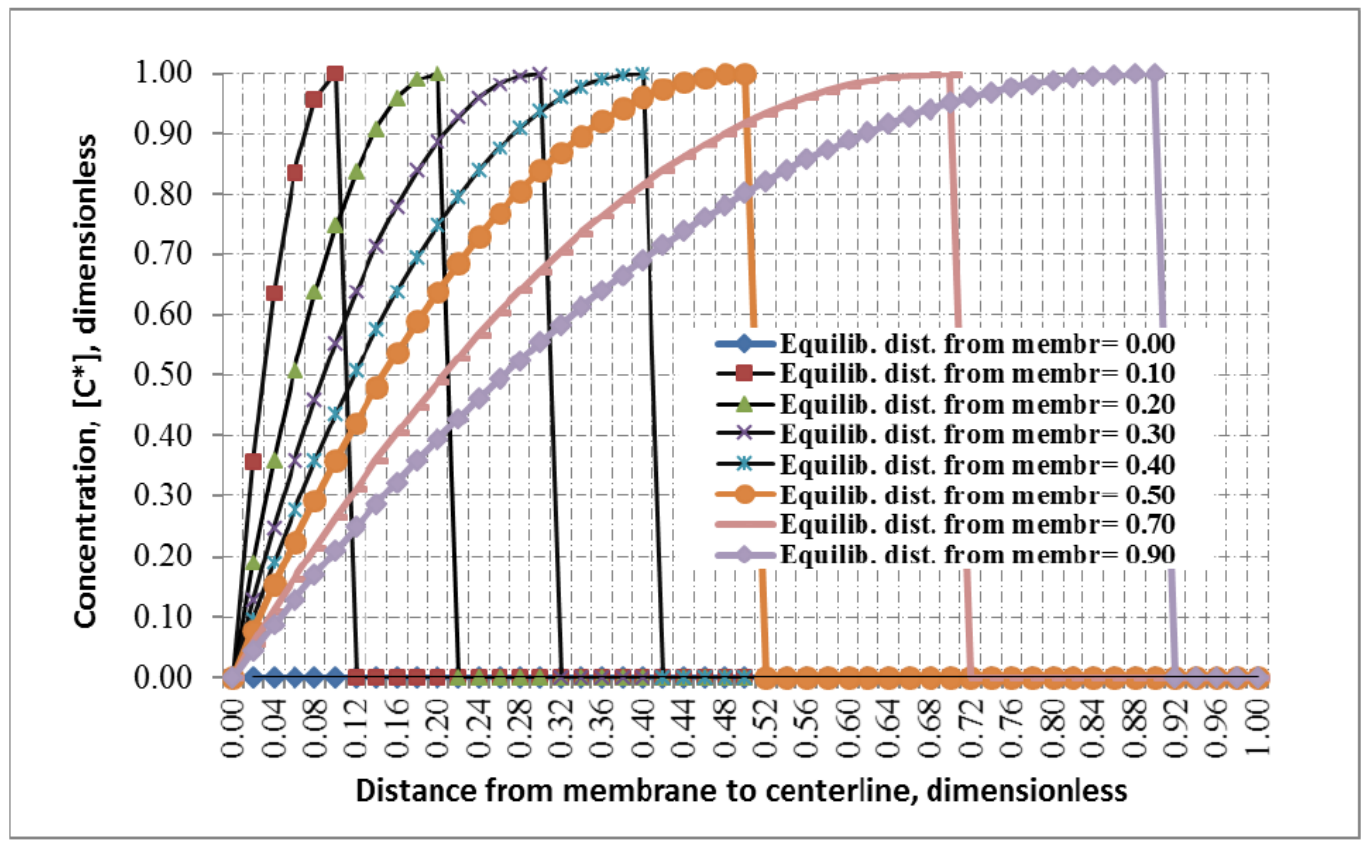

Figure 5: Dimensionless concentration profiles at different values of the upper boundary (Model).

tendency for them to be accumulated closer to membrane surface. The model calculations demonstrated that the growth of shear rate decreases surface $\mathrm{CP}$ and increases rate of migration towards centerline. Since the model represents a quantitative relation between different variables such as shear stress, transverse velocity and particle- size distribution it can be imbedded into the algorithms of different configuration.

(A) The model can estimate an impact of the radius of individual fraction on the fouling probability at existing shear stress and transverse velocity. Since the surface fouling can be interpreted as the case when the coordinate of the upper boundary of migration zone is located at the membrane surface, $\theta_{\text {crit }}=1$ that takes place at $F_{\text {shear }(\theta=1)}=F_{\text {drag }(\theta=1)}$, it implies that non-dimensional ratio- $f(R) \approx F_{\text {shear }(\theta=1)} / F_{\operatorname{drag}(\theta=1)}$ can be interpreted as a measure of fouling probability for individual fraction. Using Eqs. (14) and (17) it gives $f(R) \approx R\left[\dot{\gamma}_{M A X(\theta=1)} / V_{M A X(\theta=1)}\right]$;

(B) The model can estimate an impact of hydrodynamic conditions (such as shear stress and transverse velocity) on the probability of fouling caused by the fraction with individual radius. In order to prevent the accumulation of certain $R$-fraction at the surface the shear rate should exceed the threshold value specific for this fraction $\dot{\gamma}_{M A X(\theta=1)} \geq f(R) V_{M A X(\theta=1)} / R$;
(C) The model can be applied for the analysis of the concentration distribution (and CP degree) of individual fraction with current radius in polydisperse systems. Since any individual fraction is characterized by its individual migrating behavior and individual distribution curve depending upon the profile of shear rate. Any poly-disperse system can be represented as a combination of multiple fractions with individual parameters and specific migrating behavior. Concentration distribution (and the degree of concentration polarization) cannot be expressed based on traditional techniques in terms of mass concentration in this case. Data generated by the model can be recommended for the synthesis of optimized structure of regeneration cycle as well.

\section{ACKNOWLEDGEMENTS}

The author is thankful to the National Energy and Water Research Center (ADWEA) for support and encouragement.

\section{ABBREVIATIONS}

$C=$ concentration, $\mathrm{m}-3$

$F=$ force acting the particle, $\mathrm{N} / \mathrm{m} 2$

$f=$ dimensionless $f(R) \approx F_{\text {shear }(\theta=1)} / F_{\text {drag }(\theta=1)}$

$r_{j}, R=$ radius of individual fractions, $\mathrm{m}$ 


$$
\begin{aligned}
& H=\text { distance from membrane to centerline, } \mathrm{m} \\
& V=\text { transverse velocity, } \mathrm{m} / \mathrm{s} \\
& U, u=\text { longitudinal velocity, } \mathrm{m} / \mathrm{s} \\
& W, w=\text { velocity of back migration (relative to } \\
& Z \quad=\text { distance from membrane surface, } \mathrm{m} \\
& \dot{\gamma} \quad=\text { shear rate } \dot{\gamma}(\theta)=d u(\theta) / d \theta, \mathrm{s}-1 \\
& \tau \quad=\text { shear stress } \tau(\theta)=-\mu(d u / d \theta), \mathrm{N} / \mathrm{m} 2 \\
& \theta \quad=\text { dimensionless distance variable } \theta=1-z / H \\
& \mu \quad=\text { dynamic viscosity, } \mathrm{Pa} \mathrm{s} ;
\end{aligned}
$$

\section{Subscripts}

$$
\begin{aligned}
\min = & \text { minimum } \\
\max = & \text { maximum } \\
\text { membr = } & \text { membrane } \\
\text { crit }= & \text { critical (critical distance, } \\
& \left.\theta_{\text {crit }}=\sqrt{3-4 \dot{\gamma}_{\max } R / 3 V_{\max }}\right)
\end{aligned}
$$

\section{REFERENCES}

[1] Zydney AL, Colton CK. Concentration polarization model for filtrate flux in cross flow microfiltration of particulate suspensions. Chem. Eng. Comm. 1986; 47: 1-21. http://dx.doi.org/10.1080/00986448608911751

[2] Zydney AL, Colton CK. Fundamental studies and design analysis for cross flow membrane plasmapheresis. In: J. D. Andrade et al., editors. Proceedings of International Symposium on Artificial Organs, Biomedical Engineering and Transplantation, New York, VCH Publishers, 1987: p. 343358.

[3] Davis $\mathrm{RH}$, Leighton $\mathrm{DH}$. Shear-induced transport of a particles layer along a porous wall. Chem Eng Sci 1987; 42: 275-281.

http://dx.doi.org/10.1016/0009-2509(87)85057-1

[4] Romero CA, Davis RH. Global model of cross-flow microfiltration based on hydrodynamic particle diffusion. Journal of Membrane Science. 1988; 39: 157-185. http://dx.doi.org/10.1016/S0376-7388(00)80987-4

[5] Romero CA, and RH Davis. Transient model of cross-flow microfiltration Chemical Engineering Science 1990; 45: 1325. http://dx.doi.org/10.1016/0009-2509(90)87076-5

[6] Romero CA, Davis RH. Experimental verification of the shear-induced hydrodynamic diffusion model of cross-flow microfiltration. Journal of Membrane Science 1991; 62: 249273.

http://dx.doi.org/10.1016/0376-7388(91)80042-5

[7] Davis $\mathrm{RH}$, Sherwood JD. A similarity solution for steady-state cross-flow microfiltration. Chemical Engineering Science 1990; 45: 3203-3209.

http://dx.doi.org/10.1016/0009-2509(90)80212-W
[8] Hoek Eric MV, Kim AS, Elimelech M. Influence of cross-flow membrane filter geometry and shear rate on colloidal fouling in reverse osmosis and nano-filtration separation. Environmental Engineering Science 2002; 19: 357-372. http://dx.doi.org/10.1089/109287502320963364

[9] Mondor M, Moresoli C. Shear-induced hydrodynamic diffusion model for cross-flow microfiltration: role of the particle volume fraction. Desalination 2002; 145: 123-128. http://dx.doi.org/10.1016/S0011-9164(02)00396-X

[10] Kromkamp J, et al. Shear-induced diffusion model for microfiltration of poly-disperse suspensions, Desalination 2002; 146: 63-68. http://dx.doi.org/10.1016/S0011-9164(02)00489-7

[11] Breedveld van den Ende VD, Bosscher M, Jongchaap RJJ, Mellema J. Measurement of the full shear-induced selfdiffusion tensor of non-colloidal suspensions, Journal of Chemical Physics 2002; 116: 10529-10535. http://dx.doi.org/10.1063/1.1478770

Bian R, Yamamoto $\mathrm{K}$, Watanabe $\mathrm{Y}$. The effect of shear rate on controlling the concentration polarization and membrane fouling. Desalination 2000; 131: 225-236.

http://dx.doi.org/10.1016/S0011-9164(00)90021-3

[13] Kromkamp J, Bastiaanse A, Swarts J, Brans G, R. van Sman GM, Boom RM. A suspension flow model for hydrodynamics and concentration polarization in cross flow micro filtration. Journal Membrane Science 2005; 253: 67-79. http://dx.doi.org/10.1016/j.memsci.2004.12.028

[14] Belfort G, Davis RH, Zydney AL. The behavior of suspensions and macromolecular solutions in cross-flow microfiltration Journal of Membrane Science 1994, 96: 1-58. http://dx.doi.org/10.1016/0376-7388(94)00119-7

[15] Green G, Belfort G. Fouling of ultrafiltration membranes: Lateral migration and the particle trajectory model. Desalination. 1980; 35: 129-147. http://dx.doi.org/10.1016/S0011-9164(00)88607-5

[16] van Reis R, Zydney A. Bioprocess membrane technology. Journal of Membrane Science 2007; 297: 16-50. http://dx.doi.org/10.1016/j.memsci.2007.02.045

[17] Jiang T, Kennedy MD, Chang Kyoo Yoo, Nopens I, van der Meer W, Futselaar H, Schippers JC, Vanrolleghem PA. Controlling submicron particle deposition in a side-stream membrane bioreactor: A theoretical hydrodynamic modeling approach incorporating energy consumption. Journal of Membrane Science 2007; 297: 141-151. http://dx.doi.org/10.1016/j.memsci.2007.03.033

[18] Ould-Dris A, Jaffrin MY, Si-Hassen D, Neggaz Y. Effect of cake thickness and particle poly-dispersity on prediction of permeate flux in microfiltration of particulate suspensions by a hydrodynamic diffusion model. Journal of Membrane Science 2000; 164: 211-227. http://dx.doi.org/10.1016/S0376-7388(99)00194-5

[19] Li H, Fane AG, Coster HG, Vigneswaran S. Direct observation of particle deposition on the membrane during cross flow micro filtration. Journal of Membrane Science 1998; 149: 83-97. http://dx.doi.org/10.1016/S0376-7388(98)00181-1

[20] Mung-man Kim, Zydney A. Theoretical analysis of particle trajectories and sieving in two-dimensional cross-flow filtration system. Journal of Membrane Science 2006; 281: 666-675.

http://dx.doi.org/10.1016/..memsci.2006.04.037

[21] Bachini P, Aimar P, Feld RW. Critical and sustainable fluxes: Theory, experiments and applications. Journal of Membrane Science 2006; 281: 42-69. http://dx.doi.org/10.1016/j.memsci.2006.04.014

[22] Lifia MY, Le-Cheh $P$, Stuetz RM, Vicki Chen. Characterisation of membrane fouling deposition and removal by direct observation technique. Journal of Membrane Science 2009; 341: 163-171. http://dx.doi.org/10.1016/j.memsci.2009.06.001 
[23] Thorsen T. Concentration polarization by natural organic matter (NOM) in NF and UF. Journal of Membrane Science 2004, 233: 79-91.

http://dx.doi.org/10.1016/j.memsci.2004.01.003

[24] Zhang YP, Fane AG, Law AWK. Critical flux and particle deposition of bidisperse suspensions during cross-flow microfiltration. Journal of Membrane Science 2006; 282: 189197.

http://dx.doi.org/10.1016/j.memsci.2006.05.022

[25] Ghidossi R, Veyret D, Moulin P. Computational fluid dynamics applied to membranes: State of the art and opportunities. Chemical Engineering and Processing 2006; 45: 437-454.

http://dx.doi.org/10.1016/j.cep.2005.11.002

[26] Agashichev SP. Pressure Driven Membrane Processes (Modeling and Analysis). New York: Nova Science Publishers 2012. ISBN: 978-1-61942-411-1.

[27] Berman AS. Laminar flow in channels with porous walls, Journal of Applied Physics 1953; 24: 1232-1235. http://dx.doi.org/10.1063/1.1721476 